\title{
Shifting pedagogical priorities in facilitating mixed methods research including postgraduate student's reflections.
}

\author{
Dr Jo Gilmartin ${ }^{1}$ \\ School of Healthcare \\ University of Leeds \\ United Kingdom \\ j.gilmartin@leeds.ac.uk
}

\author{
Dr Philip Esterhuizen² \\ School of Healthcare \\ University of Leeds \\ United Kingdom \\ p.esterhuizen@leeds.ac.uk
}

Abstract-Teaching mixed methods research for quality improvement and translating evidence base practice to address meaningful change has become increasingly popular. In times of evidence-based and data-driven calls for improvement it is important to provide students with knowledge and skills about mixed methods research that will help with translation of research into practice. The literature identifies diverse pedagogical challenges that students face within mixed methods classrooms. Students are not usually equipped in both qualitative and quantitative approaches. The knowledge gap can result in deep pedagogical challenges compromising student's ability to fully understand the mixed methods paradigm. Although we are shifting from a teacher centred (pedagogical) to a learner centred, self-directed approach (andragogical) student reluctance to engage with blackboard activities and critical appraisal of mixed methods design and papers persists.

The present study explores the student perception about learning mixed methods research by collecting written reflections at the end of a mixed methods post graduate study day and across a post graduate module on mixed methods research. A reflective teaching-learning methodology was employed aligned to the self-inquiry model. Using a systematic framework for content data analysis, two distinct categories were revealed. These relate to 'barriers' and 'enablers' that influenced the quality of the learning event. This yielded four themes including 'grappling with mixed methods research', 'classroom challenges' moving to 'creative engagement with mixed methods' and 'pedagogic teacher -student interactions'. To tackle the myriad of challenges encountered, innovative teaching strategies and the sustainability of student centred approaches will be considered to lever up and empower the learning climate in mixed methods classrooms and build a pedagogical culture.

Keywords-mixed methods research, pedagogical challenges, student reflections, innovative teaching strategies.

\section{INTRODUCTION}

The need for mixed methods research to address mental and behavioural health issues has led to an increase in mixed methods studies funded by the United Kingdom (UK) Medical Research Council and the United States of America (USA)

National Institute of Health. This shift has identified the need for drawing on trans-disciplinary as well as methodological expertise; especially within the fields of nursing and health sciences for addressing complex societal issues and health problems [1-2]. As the use of mixed methods masters and $\mathrm{PhD}$ dissertations and published studies also increases, more post graduate students are interested in learning mixed methods [3]. This popularization has increased the demand for instruction in mixed methodology. Despite the increasing demand for mixed methods modules, the opportunities to learn mixed methods at a graduate level are limited relative to learning opportunities in quantitative and qualitative research. Addressing this issue, a mixed methods module and study days were integrated into the curriculum for our health science post graduate students. The purpose of our paper is to summarize the student's reflections, pedagogical challenges and prospects.

\section{BACKGROUND KNOWLEDGE}

There is a growing realisation that in our whole approach to nurse and health professional's education that contemporary pedagogies are being recognised as empowering [4]. These pedagogies are more student centred and value dialogue, diverse interpretation of information and critiquing knowledge as crucial elements. Although the importance of teacher learner dialogue and negotiation are vital characteristics, the how-to's of doing mixed methods research and the nuts and bolts of designing mixed methods studies can pose thorny challenges in the classroom context that are frequently downplayed. Fortunately, an immense contemporary literature review points to the insufficiency of pedagogical research on the challenges students face regarding learning of research methods [5].

Nonetheless in charting our course, it is vital that we make critical use of the small body of existing evidence regarding the pedagogical challenges that students face in mixed methods classrooms. A ground breaking study on the pedagogical state of teaching research methods in the UK higher education offer an in-depth critique of twenty four papers that consider research methods 'pedagogy culture'[6]. In analysing the content of these papers, they discerned three important elements of

DOI: $10.5176 / 2345-7198 \_5.1 .8$ 
effective pedagogical process [6]. First, is engaging students in a range of learning exercises/activities across the entire research process. Second, is to encourage students to carry out their own research project. Third, is to invite students to critically reflect on their own research practice and learning. Beyond this point, other writers also point to the importance of reflection in mixed methods classrooms encouraging teachers to develop an interactive reflective model, early on and throughout the entire course. This process will uncover challenges, dilemmas and confusion that students encounter and learning issues begin to be addressed, opening up the doors to creative learning [7].

We ourselves (JG \&PE) teach two graduate modules that relate to mixed methods research inquiry. Of those, one module specifically focuses on mixed methods (small heterogeneous group) and the other is a research module (large heterogeneous group) that includes one mixed methods study day. We encourage students to reflect on their experiences in learning about mixing methods and any concern or issues they may have. Addressing issues or challenges at the beginning of each study day.

The majority of the graduate students undertaking the mixed methods seminars are undertaking master's programmes and are usually at the pre-dissertation or dissertation proposal stage. They come from different professional backgrounds including nursing, midwifery, pharmacy, social work and counselling and are getting an advanced degree in the healthcare field. As teaching progressed, it became clear to faculty members that most students taking the mixed methods module had an education in one type of method, either in quantative or qualitative that were framed as a priority within their discipline. Students' background knowledge can trigger the romanticizing or treating one paradigmatic stance as strong; raising concerns about paradigm incompatibility rather than grappling with merging quant and qual paradigms. Against this background, this papers reports from a small-scale reflective inquiry on postgraduate student's perceptions of mixed methods research.

\section{PRESENT STUDY}

The main reflective data reported in this paper were obtained from 56 post graduate students who volunteered to participate. This included a convenience sample of male and female students (age range 22 to 45 years) who were undertaking the mixed method module or mixed method study day as part of a research methods module. Initially students were consulted on the first study at a face-to-face level and asked to keep written $\log$ /individual reflections of the learning process/experience and to submit on the final study day. There were, then, 56 independent sources of reflective evidence. A systematic framework was used to analyse the qualitative data and text were analysed for patterned responses and meaningful information. Data were analysed thematically.
Each reflective log/diary was read and reread by the lead researcher to develop a thematic coding scheme. The researcher (JG) initially carried out a familiarisation analysis, reading and re-reading the data and noting initial ideas. Codes were developing using both open and selective coding processes in a systematic fashion across the entire data set, collating data relevant to each code. Examples of the codes and selected content were then read by another researcher (PE), confirming or otherwise the relevance of the codes for the data. The researchers discussed the codes, considering links between the codes with a view to form a set of overall themes. After a number of iterations, four final themes emerged, two for major barriers and two for major enablers.

\section{V.MAJOR BARRIERS}

\section{A. Grappling with mixed methods}

This over-arching theme focuses on remarkable difficulties about learning mixed methods that most seem to avow. Most implied that the language and terminology used was challenging such as 'worldview', 'pragmatism', 'mixed methods', and 'multi-methods'. Others reported that mixing paradigms brought puzzlement because their own methods of training gave them little insight of how quantitative and qualitative methods were linked to a set of philosophical assumptions about the nature of the social world. Many found classroom sessions that unpacked different paradigmatic stances, for knowledge building difficult and brought confusion; especially regarding whether paradigms could be mixed within one study. For example, one pharmacist graduate with a scientific and quantitative background, explained:

'Scientific positivism is my field and I cannot accommodate interviews, voices or narratives in my research endeavours.'

Another counselling student with a robust background in qualitative research methodology, asserted:

'I'm only interested in research techniques that portray the voices, opinions and ideas of lived experiences of mental health issues. I'm biased and it is a great privilege to work with vulnerable populations and quantative measures do not fit with my paradigm.'

One nursing student undertaking a leadership programme mentioned concern regarding paradigm incompatibility and how it is possible to exist within both paradigms if they are so disparate, how to mix these paradigms to conduct a mixed methods studies. Also, other nursing students on an advanced practice master's programme mentioned that timing such as concurrent or sequential and design choice were challenging to ensure that critical ingredients can bring complementary strengths not overlapping weaknesses.

A further source of difficulty pointed to curiosity about paradigms fitting when specifying a research question to facilitate the conducting a mixed methods project. A female counselling student mentioned that autoethnography, personal narrative and reflexivity was her approach and stated:

'I'm familiar with the interpretative paradigm and I am struggling with identifying a mixed methods research question to merge qualitative and quantitative paradigms'-seems impossible'.

Several students highlighted concerns about how they should tackle analysing mixed methods data. The majority with a scientific background had undergone some statistical analysis 
training sessions but had not taken a more advanced statistical module or study days. Of the students with a qualitative background, most had undertaken an introductory module on qualitative research or had pursued workshops on phenomenology or grounded theory or facilitating focus groups but did not have much personal experience. It seemed challenging for them to position data collection methods and analytical tools in terms of their research proposals or projects. Most reported bias towards the scientific or interpretative paradigm with little understanding of the fundamentals of the research process. One social worker student reported:

'I'm very interested in child and adolescence mental health and like the case study approach but connecting objectivist quantitative strands seems very challenging to fit with a mixed methods research question'.

\section{B. Classroom challenges}

Another core theme alluded to students perceptions of the challenges posed by didactic-experiential interplay in the classroom context. Although some students reported a preference for traditional teaching methods, many confessed to feeling confused about the mixed methods language and concepts portrayed. The first study day introduces students to a range of new terms and paradigmatic stances, worldviews and questions that often surge from a given paradigmatic stance. Whilst portraying paradigmatic assumptions, we draw the discussion to particular type of research questions students' may consider in their own research projects and attempt to sketch out distinctions between confirmatory versus exploratory questions and progress to mixed methods inquiry. The latter method of inquiry often leads to confusion; especially in the development of mixed methods research questions. Some students said that they would prefer if the teacher/facilitator would dictate how exactly each mixed methods question is positioned in the research project. A pharmacist student asserted:

'The power point slides that show the decision tree for MM design with ideas for timing, weighting and mixing was difficult to understand. For me, this requires a more in-depth teacher explanation; especially outlining what type of research design would serve to answer a MM research question.

Similarly, another student on the leadership programme pointed out:

'The facilitator rushed the design lesson and I switched off and lost focus and I also felt dragged down by too much text on the slides'.

In discussing different type of mixed methods questions and designs such as sequential, concurrent or embedded we draw on case studies and a variety of mixed methods papers for students to critique. Some students portrayed a strong sense of reluctance to engage with experiential activities and felt that critiquing research papers was a very challenging task. A social work student explained:

'Critiquing lengthy mixed methods studies was difficult and using evaluation tools was hard. My classmates also seem to struggle with engagement in critiquing and discussing papers in small group work'.

Other students alluded to the challenges posed by homework activities such as critiquing research papers or developing a mixed methods research proposal feeling isolated and lonely. A physiotherapist student reported:
'Homework activities are very hard because I felt lonely and isolated and did not engage with other students online because I lacked confidence in my insights and do not understand convergence and validity in MM studies'

As these extracts show, critiquing mixed methods studies and engaging with experiential tasks was framed as difficult, being perceived as a salient feature that impacted the learning climate.

\section{MAJOR ENABLERS}

\section{Creative engagement with mixed methods}

This particular overarching theme relates to students' positive envisioning of the mixed methods research process and reports important features that help them thrive in the classroom. In contrast to the resisters, most had undertaken pre-modular reading or had been involved in mixed methods projects in their own research endeavours. Moreover from the beginning of the module, they displayed striking domains of engagement ranging from reflexive consideration of the social context of inquiry to a profound appreciation of triangulated designs. Several portrayed a respect and appreciation for methodological, methods, analytical and interpretative differences as being crucial to robust mixed methods praxis. They seemed to set a high premium of coming out of their own theoretical comfort zones and took on multiple standpoints and negotiated different research designs simultaneously. One podiatrist student asserted:

'The mixed methods language and diverse range of designs really excites me. Small group work opens doors to interrogation of new terms and the potential of mixed methods designs.'

Other students showed a marked interest in classroom dynamics, in the majority of cases viewing the experiential approach as a technology for facilitating student-centred learning. The centrality of the student experience was mentioned by a nutritionist post graduate student:

'I loved critiquing exemplary papers in small groups and clearing the muddy waters around design, convergence and validity issues. Also evaluating video clips and developing research questions in dyads was hugely beneficial to my learning'.

Some talked about the creative pedagogy displayed by some teachers; especially the zeal for student engagement, freedom of expression, a full recognition of student need, valuing group interaction, reflective practice and risk taking. For instance, a physiotherapist post graduate student mentioned:

'The teacher was brilliant and strongly committed to delivering student centred teaching. The classroom sessions were exciting and I learned so much....great insights into mixed methods'.

Other recounted curiosity about the diverse mixed methods designs that trigged discussion debate and further reading. A student undertaking the advanced practice programme disclosed:

'I was curious about the sequential, concurrent and embedded designs and I was passionate to discuss research questions that would tie up with the range of potential designs. I was reading mixed methods papers big time. I'm still grappling with convergence of different data sets'.

Another nursing student avowed:

'The mixed methods module has opened a whole new world of different research designs but I'm still curious about 
converging data sets and transforming data but its early days, for me.'

\section{Pedagogic teacher-student interaction}

This particular core theme portrays remarkable insights into pedagogical strategies that empowered students to engage with dialectical pluralism (DP) theory creatively. The heart of the matter is perhaps in acknowledging that student empowerment clearly opens up the learning climate and increases negotiation and bargaining power. The facilitator strives to reduce power differentials, giving student's space to chip away at the mixed method design components and at the analytical/ interpretative conundrum despite the challenges involved. In some of the reflective accounts given the patterns mainly relate to the power of experiential activities. In other instances, however, a different kind of insight is being reported: not just experiential, but 'professional growth' in the sense of working with blended learning strategies, new technology and critical teaching strategies. The strongest indications of this are in 32 cases where students reported the value of different learning experiences. For instance, a counselling student noted:

'The Padlet wall was brilliant for sharing critical appraisal comments of case study papers and helped me overcome my inhibited impulses about sharing. I just went to wall and wrote like a 'free child'. I also found the mixed methods language difficult but the small group work helped me to relax and let go the underlying dread of a new paradigm. I began to flourish.'

A further example, one nursing student recounted:

'The tutors were dynamic, creative and encouraged critical thinking bringing interpretative tensions and contradictions out in the open'. The classroom tasks were highly selfdirecting and encouraged risk taking and playfulness.'

Others celebrated the potential of small group collaboration: one $\mathrm{PhD}$ student asserted:

'The Padlet Wall was a great platform for critiquing mixed methods papers but I just find group work opens up my extremely shallow notions and sharpens my insights. Group work was spontaneous, flexible and marvellous for creative learning tasks and ideas emerge that were literally unthinkable, to me.

The success stories celebrate afresh the benefits of creative pedagogy.

\section{V1. DISCUSSION}

The findings identify the full spectrum of challenges, resistance, turning points, transitions and positive strategies and their influence on students learning. The challenges foregrounded with respect to grappling with mixed methods pointed to challenges around introducing philosophical assumptions, research typologies and methodologies for mixed methods research to students with no previous expose can possibly lead to conceptual confusion or breakthrough. Contrastingly, students can also be challenged when their pre-conceived ideas or worldviews about research are embedded in either quantitative or qualitative traditions. Learning opportunities were clearly enhanced in the creative engagement theme by using experiential approaches and creative activities; especially to examine multiple worldviews which expanded students' ways of seeing, knowing and thinking to include different and dialectical forms of knowledge. According to the study findings, the use of creative and non-linear methods of teaching resulted in students increased receptivity and engagement to new ways of perceiving knowledge, conceptualising research, and learning the theory and practice of mixed methods research dynamically. Against these positive points cited above, however, it must be said that there is a remarkable array of challenge and reluctance in the reflective evidence presented by the respondents too as cited particularly in the classroom challenges theme.

The pedagogic teacher-student interaction theme has put forward a useful range of findings already recognised in the literature as important characteristics of a positive learning climate [9]. This is in relation to the use of experiential approaches, use of blended learning and allowing students to share and explore real life clinical problems. Effective and creative facilitation appeared to trigger motivation, cooperation, collaboration and engagement of students despite the complexity when there are multiple strands in a mixed research design.

In contrast to this perception, there is remarkable evidence in the classroom challenges theme of more negative characteristics where some students seemed to be put off by the theory of dialectical pluralism and the use of a dialectical/dialogical and hermeneutical approach to learning. They seemed to find the non-linear journey de-motivating, with a lack of curiosity and engagement. The barriers identified by students such as reluctance to engage with learning activities influence the learning climate negatively and need to be addressed with positive teaching strategies that enhance mixed research pedagogy.

The rapid increase in Internet access and huge advances in online technology in recent years has enabled us to consider more creative ways to teach in the context of higher education. This pedagogical area is ripe for integrating into learning mixed methods is the inclusion of articulate presentations or webinars and You-Tube clips that cover a range of mixed methods topics. Of course the quality and validity of such resources needs critical appraisal but they offer a robust contribution to students in this inquiry who struggle with mixed methods language and new paradigms.

Moreover the flipped classroom model or inverted classroom has developed as an optimistic alternative to lecture-based teaching and it puts forward a useful framework for integrating online learning technologies with active, dynamic and collaborative learning [10-11]. This has the potential for engaging resisters and building a mixed methods pedagogy culture too. This particular approach allows students to work independently outside the classroom, at their own pace and promotes active learning and greater collaborative application with peer and facilitator support. This collaborative and interactional theories of flipped learning are embedded in 
Bloom's revised taxonomy of thinking and learning [12]. Hence, the flipped classroom allows higher and lower orders of cognitive work including knowledge application, analysis, synthesis and deep learning [13].

In the flipped classroom instructional model, the self-directed learning activities, undertaken independently outside the classroom session are used in the classroom context to deepen the acquisition of knowledge. Having set the tone for discussion through perhaps posting on the Padlet wall (www.padlet.com), the comments can be discussed in a more personalised learning environment where students might be more comfortable to engage collaboratively after having had the opportunity to think through their peers' comments. This approach has the potential to engage the resisters mentioned previously and foster more open dialogue in small group discussion, with the lecturer facilitating the discussion and helping to clarify possible misunderstandings. This type of hands on approach affords students opportunity to work through the thorny issues about learning mixed methods research with peer support. Studies in public health and among medical students using flipped pedagogy also report positive and encouraging results [14-15].

In class creative exercises that specifically engage students with different ways qualitative and quantitative findings connect or inform one another and walking through the steps researchers took to complete their mixed methods project or published papers could open up the learning climate. This ties up to the grappling with mixed methods theme that requires creative pedagogy. A novel pedagogy strategy to consider is Open Space Learning (OSL), a creative emerging paradigm of learning informed by psychology and neuroscience and mostly, the theoretical approach of Vygotsky [16] and experiential learning [17]. Epistemologically conceptualizing knowledge as 'unfinished', the process encourages learners to holistically navigate the unknown through body and mind [18]. It is a principle of the OSL methodology that space is an important factor in the quality of the learning event [18].

Interestingly space is defined pedagogically by how learners place themselves within it, and this sense should be considered philosophically as well as physically. In an OSL context it is important to open public space as well as probate space in which students learn. Crucially, experience involves risk taking, experiment and not knowing the outcomes of particular area of exploration, but being willing to take the opportunity that the opening of a space affords [18]. Students could explore and act out mixed methods terminology, study designs such as sequential, concurrent, embedded and transformative or explore integration/synthesis and legitimation issues. Open space learning could enable great opportunity for students to experiment in new and creative ways to lever up knowledge and insight and build up a mixed methods pedagogy culture as well.
Furthermore the less hierarchical use of space encourages collaborative learning through experience which is a cognitive process but also triggers affective, physical and interpersonal [19]. With knowledge production taking a more fluid collaborative form of existence, implications are generated for the power dynamics of educational settings; especially mixed methods research.

In an attempt to probe dialectical pluralism (DP) theory and practice further and problems with student engagement, TeamBased Learning (TBL) is another strategy to consider. This approach has gained substantial popularity in higher education and enables health professional educators to provide students with the real experience of working in small teams to solve 'authentic clinical problems' [20-21]. TBL has different formats or units and emphasis three major phases. Phase one involves pre-class individual study with clear student objectives using blended technology or other blackboard activities. The second phase is associated with a Readiness Assurance Process (RAP) where the individual student and team's understanding and knowledge is determined through a Readiness Assurance Test (RAT). Throughout phase three, students use concepts tested in phase two to solve clinical cases or scenarios or perhaps design a mixed method study in response to a clinical problem [21]. The challenges of implementing TBL and effort to prepare the materials are well articulated by a recent UK study [22].

At the very least, there is evidence to suggest that TBL empowers class engagement and teamwork values with a corresponding growth in learning, exam scores and academic achievement [20, 23]. Nonetheless, a contemporary systematic review examining the effectiveness of blended technology with TBL reported limited evidence that blended TBL improved student outcomes and further research is required [24].

In conclusion, creative pedagogy has the potential to be a great contribution to facilitating a mixed methods pedagogy culture. These particular approaches provide students with opportunity to share their own learning strategies whilst grappling with the slippery terminology and processes that surrounds the mixed method research paradigm. Mixed methods research continues to impact and be impacted by patterns of knowledge production relating to healthcare practice and professional discourse. There is no doubt that educators need to invest in developing strategies for engaging students with research based subjects more powerfully. The empirical data suggest that flipped learning, open space learning and team based learning might lead to increased student learning, engagement and substantively on a deeper level. A meta-analysis has provided insight into the effectiveness of within-class grouping on student academic achievement [25]. However further research is necessary to understand the impact of the 
creative teaching and learning strategies within post graduate education.

\section{ACKNOWLEDGEMENT}

Jo Gilmartin and Philip Esterhuizen extend a special thanks to all the postgraduate students who contributed to the reflect inquiry.

\section{REFERENCES}

[1] Creswell J.W. A concise introduction to Mixed Methods Research.2015a. Thousand Oaks, CA: Sage.

[2] Doyle L, Brady A.M.,\& Byrne G. An overview of mixed methods research-revisited.Journal of Research in Nursing. 2016. 21(8) 623-635.

[3] Creswell, J.W. Planto Clark, V.L. Designing and conducting mixed methods research. $2^{\text {nd }}$ edition. 2011.Los Angeles, CA: Sage Publications.

[4] Horsfall, J., Cleary, M., Hunt, G.E. Developing a pedagogy for nursing teaching-learning. Nurse Education Today. 2012.32, 930-933.

[5] Earley, M. A synthesis of the literature on research methods education. Teaching in Higher Education. 2014. 19, 242-253.

[6] Kilburn, D., Nind, M.,Wiles,R. Learning as rearchers and teachers: The development of a pedagogical culture for social science research methods? British Journal of Educational Studies.

2015. 62, 191-207.

[7] Hess-Biber, S. The problems and prospects in the teaching of mixed methods research. International Journal of Social Research Methodology. 2015.18 (5), 463-477.

[8] Braun, V., Clark, V. Successful qualitative research: a practical guide for beginners.2013. London, Sage Publications.

[9] Onwegbuzie AJ., Frels RK., Leech NL., Collins KMT. A mixed methods study of pedagogical approaches and student learning in doctoral-level mixed research courses [Special Issue]. International Journal of Multi Research Approaches. 2011, 5, 169202.

[10] Bergam J, Sams A. 2012. Flip your classroom: Reach every student in every class. Report, International Society for Technology in Education, Arlington, VA.

[11] Bishop J, Verleger M. 2013. The Flipped Classroom: A survey of the Research Paper presented at $120^{\text {th }}$ ASEE Annual Conference \& Exposition , 23-26 June, Altanta, American Society for Engineering Education.

[12] Bloom B, Engelhart M, Furst E, Hill W, Krathwohl
D. Taxomy of Educational Objectives: The Classification of Educational Goals: Handbook 1: Cognitive Domain. 1956. New York David McKay. [13]Anderson LW, Krathwohl DR, Bloom BS. A

Taxonomy for Learning, Teaching and Assessing. 2005. New York, Longman.

[14] MeEvoy CS, Cantore KM, Denlinger LN, Schleich MA, Stevens NM, Swavely SC, Odom AA, Novick MB. Use of medical students in a flipped classroom programme in nutrition education for fourth grade school students. 2016. Health Education Journal, 75 (1), 38-46.

[15]Galway LP, Corbett KT, Takaro TK, Tairyan K, Frank E. A novel integration of online and flipped classroom instructional models in public health higher education. 2014. BMC Medical Education. http://www.biomedcentral.com/1472-6920/14/181.

[16] Daniels H. ed Vygotsky and Pedagogy. 2001. London \& New York, Routledge.

[17]Kolb DA. Experiential Learning: experience as the source of learning and development.1984. Englewood Cliffs, NJ: Prentice Hall.

[18]Monk N, Chillington Rutter C, Neelands J. Heron J. Open Space Learning: A study in Trans-disciplinary Pedagogy. 2011. London: Bloomsbury.

[19]Savin-Baden M. Learning Spaces: Creating opportunities for knowledge creation in academic life. 2008.Maidenhead, England: New York: McGraw Hill.

[20] Cheng CY, Lion SR, Tsai HM, Chang CH. The effects of team based learning on learning behaviour in the maternal-child nursing course. 2014. Nurse Education Today 34, 23-30.

[21]Parmelee D, Michaelsen L, Cook S, Hudes P. Team based learning a practical guide: 2012 AMEE guide no 65.Med Tech 34, e275-e 287.

[22]Morris J. Implementing of a team-based learning course: Work required and perception of the teaching team. 2016. Nurse Education Today, 46, 146-150.

[23]Morris J. The use of team-based learning in a second year undergraduate pre-registration nursing course on evidenced-informed decision making. Nurse Education in Practice. 2016, 21, 23-28.

[24] River J, Currie J, Crawford T, Betihavas V, Rndell S. A systematic review examining the effectiveness of blended technology with team based learning. 2016. Nurse Education Today, 45, 185-192.

[25] Lou Y, Abrami PC, Spence JC. Poulsen C, Chambers B, d'Apollonia S. Within-Class Grouping: A MetaAnalysis. Review of Educational Research 1996, 66 (4), 423-458. 
Dr Jo Gilmartin is a lecturer at the University of Leeds, U K. Her research interests include body image, obesity and creative pedagogy in contemporary classrooms in higher education.

Dr Philip Esterhuizen is a Associate Director of Student Education: Curriculum and Assessment at the School of Healthcare, University of Leeds. His research interests include pedagogy and student achievement. 